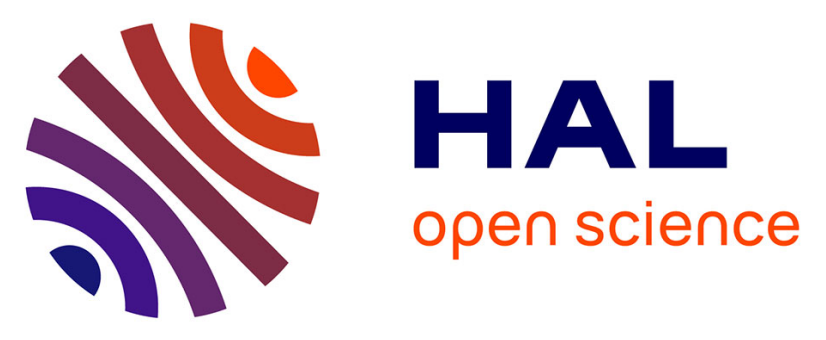

\title{
Identification of apolipoprotein C-III as a potential plasmatic biomarker associated with the resolution of hepatitis $\mathrm{C}$ virus infection
}

Sonia Molina, Dorothée Missé, Stéphane Roche, Stéphanie Badiou, Jean-Paul

Cristol, Claude Cb Bonfils, Jean-François Dierick, Francisco Veas, Thierry

Levayer, Dominique Bonnefont-Rousselot, et al.

\section{To cite this version:}

Sonia Molina, Dorothée Missé, Stéphane Roche, Stéphanie Badiou, Jean-Paul Cristol, et al.. Identification of apolipoprotein C-III as a potential plasmatic biomarker associated with the resolution of hepatitis C virus infection. PROTEOMICS - Clinical Applications, 2008, 2 (5), pp.751 - 761. 10.1002/prca.200800020 . hal-01662783

\section{HAL Id: hal-01662783 \\ https://hal-amu.archives-ouvertes.fr/hal-01662783}

Submitted on 13 Dec 2017

HAL is a multi-disciplinary open access archive for the deposit and dissemination of scientific research documents, whether they are published or not. The documents may come from teaching and research institutions in France or abroad, or from public or private research centers.
L'archive ouverte pluridisciplinaire HAL, est destinée au dépôt et à la diffusion de documents scientifiques de niveau recherche, publiés ou non, émanant des établissements d'enseignement et de recherche français ou étrangers, des laboratoires publics ou privés. 


\title{
Identification of apolipoprotein C-III as a potential plasmatic biomarker associated with the resolution of hepatitis $\mathrm{C}$ virus infection
}

\author{
Sonia Molina 1, 2 , Dorothée Missé3 ${ }^{3}$, Stéphane Roche ${ }^{4}$, Stéphanie Badiou ${ }^{5}$, \\ Jean-Paul Cristol ${ }^{5}$, Claude Bonfils ${ }^{6}$, Jean-François Dierick ${ }^{7}$,Francisco Veas ${ }^{8}$, \\ Thierry Levayer ${ }^{9}$, Dominique Bonnefont-Rousselot ${ }^{10}$, Patrick Maurel ${ }^{2}$, \\ Joliette Coste ${ }^{1,2}$ and Chantal Fournier-Wirth ${ }^{1,2}$ \\ ${ }^{1}$ Etablissement Français du Sang, Laboratoire de R\&D-Agents Transmissibles par Transfusion, \\ Montpellier, France \\ 2 INSERM, Physiopathologie hépathique, Montpellier, France \\ ${ }^{3}$ UMR 2724 CNRS/IRD, Génétique et évolution des maladies infectieuses, Montpellier, France \\ ${ }^{4}$ Laboratoire de Biochimie, Hôpital St Eloi, Montpellier, France \\ ${ }^{5}$ Laboratoire de Biochimie des Lipides, Hôpital Lapeyronie, Montpellier, France \\ ${ }^{6}$ CRCM, CRLC Val d'Aurelle-Paul Lamarque, Montpellier, France \\ ${ }^{7}$ BIOvallée-Proteomics, Gosselies, Belgium \\ ${ }^{8}$ IRD UR178, Immunologie Virale et Moléculaire, Montpellier, France \\ ${ }^{9}$ Etablissement Français du Sang, Laboratoire de Qualification Biologique des Dons, Montpellier, France \\ ${ }^{10}$ UF de Biochimie des maladies Métaboliques, Groupe Hospitalier Pitié-Salpêtrière, Paris, France
}

Understanding the virus-host interactions that lead to approximately $20 \%$ of patients with acute Hepatitis C Virus (HCV) infection to viral clearance is probably a key towards the development of more effective treatment and prevention strategies. Acute hepatitis C infection is usually asymptomatic and therefore rarely diagnosed. Nevertheless, HCV nucleic acid testing carried out on all blood donations detects donors who have resolved their HCV infection after seroconversion. Here we have used SELDI-TOF-MS technology to compare, at a proteomic level, plasma samples respectively from donors with HCV clearance, from donors with chronic HCV infection and from unexposed healthy donors ( $n=15$ per group). A candidate marker of about $9.4 \mathrm{kDa}$ was detected as differentially expressed in the three groups. After purification we identified by nanoLC-Q-TOF-MS/MS this candidate marker as Apolipoprotein C-III (ApoC-III). The identification was confirmed by western blot analysis. Levels of ApoC-III were then determined in the 45 plasma samples by immunoturbidimetric assay. ApoC-III was found to be higher in donors who had resolved their HCV infection than in donors with chronic infection, results which were consistent with SELDI-TOF-MS data. ApoC-III is the first reported candidate biomarker in plasma associated with the spontaneous resolution of HCV infection.

\section{Keywords:}

ApoC-III / Biomarker / HCV clearance / Plasma / SELDI-TOF-MS
Received: April 20, 2007 Revised: December 11, 2007 Accepted: January 11, 2008
Correspondence: Dr. Chantal Fournier-Wirth, Laboratoire de R\&D-Agents Transmissibles par Transfusion, Etablissement Français du Sang Pyrénées-Méditerranée, 240 avenue Emile Jeanbrau, 34094 Montpellier Cedex 5, France E-mail: chantal.fournier@efs.sante.fr

Fax: +33-4-6761-6456
Abbreviations: ApoC-III, apolipoprotein C-III; HCV, hepatitis C virus; HDL, high density lipoprotein; LDL-C, low density lipoprotein; NAT, nucleic acid testing; SRBI, scavenger receptor class B type I; TG, triglycerides; VLDL, very low density lipoprotein 


\section{Introduction}

Acute hepatitis C virus (HCV) infection is usually a mild and asymptomatic disease, making early diagnosis difficult [1]. Spontaneous resolution of HCV infection occurs in 10-30\% of acutely infected patients and is associated with a broad, specific and vigorous cellular immune response [1-4]. In 70$90 \%$ of patients, however, HCV persists and causes chronic hepatitis with its life-threatening complications, including liver failure and hepatocellular carcinoma [1]. An estimated 170 million people worldwide are chronically infected with HCV including five million in Europe [5]. Furthermore, current anti-viral therapy is expensive, relatively toxic and effective in only $50-60 \%$ of treated patients [3]. There is as yet no vaccine against $\mathrm{HCV}$ but the development of at least a partly effective vaccine seems feasible [6]. Many aspects of HCV infection remain enigmatic. A better understanding of the early stages of the HCV infection is probably a key to the development of more effective treatment and prevention strategies [4].

The systematic screening of viral markers in blood donations has decreased the risk of transfusion-associated hepatitis to a negligible level in developed countries [5]. New screening methods such as the nucleic acid testing (NAT) of blood and plasma donors has yielded important insights into the epidemiology, pathogenesis and prognosis of $\mathrm{HCV}$ infection [7, 8]. Recently, in a transfusion study, viral and host factors in early HCV infection have been studied among 94 donor-recipent pairs in which there was transmission. It appears that host factors are more important determinants of acute HCV infection dynamics than virus-associated factors [9]. Since plasma potentially contacts every cell as it circulates through the body, it may carry clues both to diagnosis and treatment of disease [10].

It is commonly expected that recent techniques leading to the detection of a growing number of trace proteins within biological fluids will result in the discovery of new biomarkers. Recent advances in proteomics associating protein separation technologies and MS have provided opportunities for biomarker identification and characterization [11]. SELDI-TOF-MS is a variant of MALDI-TOF-MS, in which the samples are directly applied to a chip coated with a specific chemical or biochemical matrice. The bound proteins are then analyzed by MS to obtain the protein fingerprint of the sample $[12,13]$. Consequently, SELDI-TOF-MS has an excellent potential for protein profiling. This method, which is suitable for the analysis of a large number of biological samples, has shown promise for biomarker discovery in HCV infection and hepatocellular carcinoma [14-18].

The aim of this project was to identify biomarkers associated with the resolution of acute HCV infection. The main difficulty encountered in comparing normal with pathological patterns is linked to the choice of the relevant samples. HCV NAT detects donors with viral clearance after seroconversion, as demonstrated both by HCV RNA negativity and $\mathrm{HCV}$ seropositivity. In consequence, we have applied
SELDI-TOF-MS to analyze the plasma from blood donors with well-defined serological and virological profiles. We have compared the plasma proteome for three groups of donors (i) individuals who cleared their HCV infection, (ii) chronic HCV carriers, and (iii) unexposed healthy individuals to investigate whether the differential expression of certain proteins could be associated with the resolution of HCV infection. Several differentially expressed protein peaks have been detected. After purification, proteins of interest were analyzed by nanoLC-Q-TOF-MS/MS. A candidate biomarker was characterized and its identity was validated by western blot analysis and immunoturbidimetric assay in all plasma samples.

\section{Materials and methods}

\subsection{Samples}

Human plasma samples $(n=45)$ were obtained from the Etablissement Franç ais du Sang Pyrénées-Méditerranée; 15 samples from blood donors who tested negative for all HCV markers, 15 samples from donors with resolved HCV infection, and 15 from donors with chronic hepatitis C. Plasma samples were aliquoted and then stored at $-80^{\circ} \mathrm{C}$ until use. None of the individuals had received an antiviral treatment prior to donation. All plasma samples were screened for antiHCV antibodies with the Ortho HCV v3.0 ELISA test system (Ortho clinical diagnostics, Raritan, NJ, USA). The RIBA HCV 3.0 immunoblot assay (Chiron, Emeryville, CA, USA) was used as the confirmatory test. Screening of HCV RNA was systematically performed by PCR using the Cobas Ampliscreen HCV v2.0 assay (Roche Molecular Systems, Branchburg, NJ, USA). We further quantified HCV RNA levels in plasma samples from donors with chronic hepatitis by the Cobas Amplicor HCV Monitor v2.0 test (Roche Molecular Systems). The clinical characteristics of donors are shown in Table 1.

\subsection{Profiling of plasma using SELDI-TOF-MS}

Optimization studies on a hydrophobic surface were carried out prior to profiling analysis. All plasma samples were albumin depleted with Cibacron Blue before analysis (Sigma, Saint Louis, Mo, USA). On H50 ProteinChip arrays, optimal conditions were found to be a 1:10 dilution of depleted plasma with the binding buffer $(1 \times$ PBS, $0.1 \%$ TFA). H50 arrays were pre-washed twice with $5 \mu \mathrm{L}$ of $50 \%$ ACN for $5 \mathrm{~min}$. After air drying, the surface was loaded twice with $2 \mu \mathrm{L}$ of binding buffer for $2 \mathrm{~min}$. and then $5 \mu \mathrm{L}$ of $1: 10$ diluted plasma was added to each spot and incubated for $1 \mathrm{~h}$ in a humidified chamber at room temperature. Plasma was removed and each spot was individually washed three times for 2 min with $5 \mu \mathrm{L}$ washing buffer (10\% ACN, 0.1\% TFA) followed by one quick wash with deionized water. The surface was allowed to air dry, and $1 \mu \mathrm{L}$ of SPA (Ciphergen Bio- 
systems, Palo Alto, CA, USA) in 50\% v/v ACN and 0.5\% v/v TFA was added twice to each spot. $\mathrm{H} 50$ arrays were then read in a ProteinChip Reader system, PBSIIc serie (Ciphergen Biosystems). The spectra were generated by the accumulation of 80 laser shots through the spot at a laser intensity of 180 arbitrary units. Spectra were collected and analyzed using the Ciphergen ProteinChip software (v3.0). All mass spectra were then normalized using TIC normalization before proceeding to statistical analysis. The TIC method assumes that on average, the total number of proteins being expressed is constant across the samples being normalized. The process takes the TIC used for all the spots, averages the intensity, and adjusts the intensity scales for all the spots in order to display data on the same scale. The $m / z$ ratio of each of the peaks to be quantified was determined according to externally calibrated standards (Ciphergen Biosystems). Peak clustering in the range from 1000 to $20000 \mathrm{~m} / z$ ratio was performed using Biomarker Wizard Software (Ciphergen Biosystems) at settings that provide a $0.3 \%$ mass window and $5 \% \mathrm{~S} / \mathrm{N}$ determination. Statistics were performed using the nonparametric Mann-Whitney $U$-test on the maximal intensity of each peak. Significant threshold was set at $p<0.05$.

\subsection{Enrichment of biomarkers candidates}

We performed a fractionation of the plasma proteins with increased concentrations of ammonium sulfate (Sigma) [19, 20]. This phenomenon of protein precipitation in the presence of excess salt is known as salting-out. We used increasing salt concentrations to reach 20, 40, 60, 80 and $100 \%$ saturation of ammonium sulfate as follows. Ammonium sulfate was added to $50 \mathrm{~mL}$ of undiluted plasma to reach $20 \%$ saturation. After incubation at room temperature with gentle agitation for $1 \mathrm{~h}$, the tube was then centrifuged at $11300 \times \mathrm{g}$ for $15 \mathrm{~min}$ at $20^{\circ} \mathrm{C}$. The supernatant was transferred to another tube and ammonium sulfate was added to reach $40 \%$ saturation. The mixture was treated as above. The proteins in the supernatant were further precipitated with 60,80 and $100 \%$ ammonium sulfate saturation respectively. The precipitated proteins were collected and categorized according to the concentration of the salt used for saturation. Each of the five pellets was resuspended in $2 \mathrm{~mL}$ of biomolecular grade water and then aliquoted and stored at $-80^{\circ} \mathrm{C}$ until use. The precipitated proteins were analyzed by SELDI-TOF-MS on normal phase NP20 arrays as recommended by the manufacturer in order to compare their protein profile with the original unfractionated plasma.

\subsection{Recovery of enriched proteins using passive elution}

A 1:10 diluted plasma sample from a donor with resolved HCV infection and the 1:5 diluted precipitates recovered from the precipitation steps were separated by SDS-PAGE under reducing conditions on a $12 \%$ acrylamide NuPAGE Bis-tris gel (Invitrogen, Carlsbad, CA, USA). The apparent molecular masses were determined by running the SeeBlue pre-stained protein standard (Invitrogen). Bands of molecular weight approximating to candidate biomarkers were cut out from the CBB stained gel. Passive elution was effected as previously described by Currid et al. [21]. Briefly, gel pieces were washed with $50 \%$ ACN/ $50 \mathrm{mM}$ ammonium bicarbonate and then dehydrated with $100 \%$ ACN. The gel pieces were heated to $50^{\circ} \mathrm{C}$, before the addition of a $45 \%$ formic acid/30\% ACN/10\% isopropanol solution and incubation in a sonicating waterbath for $30 \mathrm{~min}$ at room temperature. The passively eluted material was then spotted on an H50 array, followed by addition of saturated sinapinic acid.

\subsection{Identification of the proteins by nanoLC-Q-TOF- MS/MS}

The 1:5 diluted precipitate recovered from the $80 \%$ ammonium sulfate saturation was analyzed by 1 -DE under reducing conditions as described in Section 2.4. The band of interest and a blank area of the gel were excised from the CBB stained gel before in-gel tryptic digestion. The resulting peptides were analyzed by nanoflow-HPLC-Q-TOF- MS/MS using a CapLC coupled with a Q-TOF Ultima Global Instrument (Waters/Micromass UK, Manchester, UK). The MS/ MS fragment data were integrated using MASCOT (Matrix Science, Boston, MA, USA) searching the National Center for Biotechnology Information and Swiss-Prot databases. The parameters for the query were: species of origin Homo sapiens, digestion by trypsin allowing for no more than one missed cleavage, peptide mass tolerance $50 \mathrm{ppm}$, fragment mass tolerance $0.6 \mathrm{Da}$, possible charge $+1,+2$, +3 . The threshold of significance $(p<0.05)$ was given with MASCOT by a score of 55 .

\subsection{Western blot analysis}

A 1:10 diluted sample from the plasma used in the enrichment step was albumin depleted before analysis by electrophoresis under reducing conditions as described in Section 2.4. A 1:5 diluted precipitate recovered from the $80 \%$ ammonium sulfate saturation and a sample of purified human apolipoprotein C-III (ApoC-III) protein from human plasma (Chemicon, Temecula CA, USA) were used as controls. After transfer to a NC Hybond ECL membrane (GE Healthcare, Buckingamshire, UK) and immunodetection with a 1:20000 dilution of a polyclonal rabbit anti-human ApoC-III antibody (Biogenesis, Poole, UK), the rabbit IgG was then probed with 1:10000 dilution of HRP-labeled goat anti-rabbit antibodies (Sigma) and the proteins were visualized using an enhanced chemiluminescence detection method (GE Healthcare).

\subsection{Plasma lipid analysis}

ApoC-III levels were measured by immunoturbidimetric assay (Kamiya biomedical company, Seattle, USA) adapted 
on the Konepro analyzer (Thermo Electron, Cergy-Pontoise, France) with a linearity ranging from 0.03 to $0.3 \mathrm{~g} / \mathrm{L}$. ApoA-I and apoB concentrations were determined by immunonephelometric assay (Immage 800, Beckman Coulter, Villepinte, France). ApoA-II level was determined by immunonephelometry on a BN II nephelometer analyzer (Dade Behring, Marburg GmbH, Germany). ApoC-I level was determined by competitive Elisa on Vitros 950 as previously described by Dautin et al. [22]. ApoC-II and Apo-E levels were measured by immunoturbidimetric assay (Randox, Mauguio, France) adapted on the Olympus AU 640 analyser (Olympus, Rungis, France). Total cholesterol (TC), high density lipoprotein (HDL)-cholesterol, low density lipoprotein (LDL)-cholesterol, and triglycerides (TG) levels were measured in plasma samples by routine enzymatic methods on the Konepro analyzer. Very low density lipoprotein (VLDL)-cholesterol was estimated as TC-(HDL-cholesterol+LDL-cholesterol). Statistical differences between groups were determined using Wilcoxon's test (www.u707. jussieu.fr/biostatgv/index.html). Significant threshold was set at $p<0.05$.

\section{Results}

\subsection{Plasma screening on $\mathrm{H} 50$ proteinchip arrays}

For the purposes of our study, we analyzed archived plasma samples, from blood donors, which had been screened for HCV infection markers. Acute hepatitis C infection is usually asymptomatic and therefore rarely diagnosed. Nevertheless, HCV NAT, implemented on all blood donations since July 2001 in France, detects asymptomatic donors. Based on screening tests, the samples were divided into three groups (Table 1). To minimize unrelated variables, samples used in this study have been restricted with respect to sex and age. The "Negative" group $(n=15)$ represents blood donors tested negative for all HCV markers. Individuals in the "Resolved" group $(n=15)$ show evidence of resolution of HCV infection (anti-HCV antibodies (+), HCV RNA $(-))$. The "Chronic" group $(n=15)$ comprises patients with chronic HCV infection (anti-HCV antibodies (+), HCV RNA (+)). HCV RNA levels were evaluated in the last group; their values ranged from $2 \times 10^{4}$ to $>50 \times 10^{4} \mathrm{IU} / \mathrm{mL}$.

Plasma samples $(n=45)$ from the three groups (Negative, Resolved, Chronic) were albumin depleted and diluted 1:10 before analysis on six H50 arrays using SELDI-TOF-MS technology. All mass data were baseline subtracted, normalized using total ion current, and peak clusters were generated by Biomarker Wizard software. The representative profile of plasma samples is presented in Fig. 1. Four peaks were found to be differentially expressed between the three sample groups with significant $p$-values $(p<0.05)$ or very significant $p$-values $(p<0.01)$, and one peak with highly significant $p$-value $(p<0.001)(1500-20000 \mathrm{~m} / z$ ratio was our optimal range setting). The average $m / z$ values associated with these
Table 1. Characteristics of donors involved in the study

\begin{tabular}{|c|c|c|c|}
\hline Donor group ${ }^{\text {a) }}$ & Negative & Resolved & Chronic \\
\hline Sex (Male/Female) & $(7 / 8)$ & $(8 / 7)$ & $(7 / 8)$ \\
\hline Age (mean) & $36.07 \pm 14.00$ & $38.47 \pm 9.73$ & $35.60 \pm 9.77$ \\
\hline Anti HCV antibody & - & + & + \\
\hline Screening test & - & $4.91 \pm 1.57$ & $7.81 \pm 1.11$ \\
\hline Confirmatory test & - & ++ & ++++ \\
\hline HCV RNA & - & - & + \\
\hline Qualitative PCR & - & - & + \\
\hline Quantitative PCR & - & - & $2 \times 10^{4}-50 \times 10^{4} \mathrm{IU} / \mathrm{mL}$ \\
\hline
\end{tabular}

a) Negative (unexposed), Resolved and Chronic HCV infection respectively.

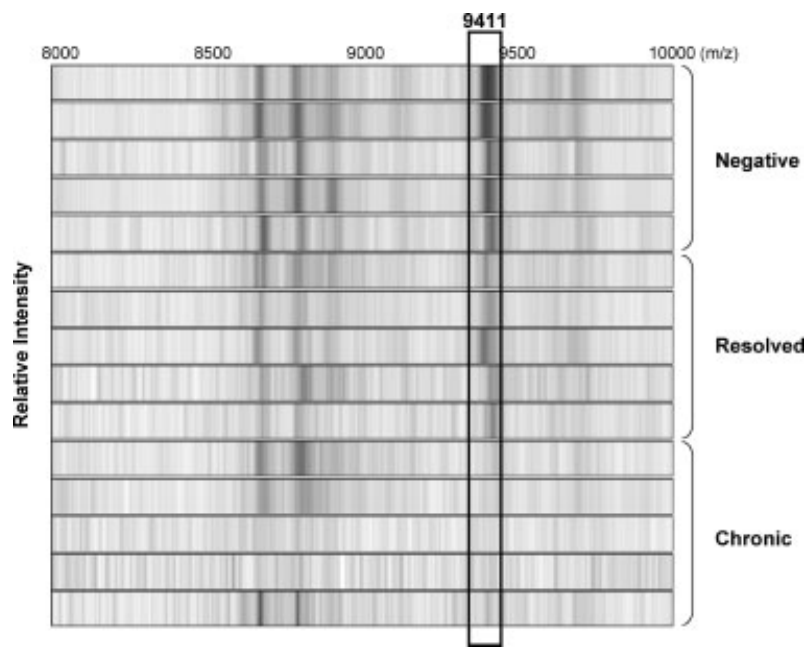

Figure 1. SELDI-TOF-MS protein spectra of human plasma samples from negative donors versus donors with resolved or chronic HCV infection (five representatives out of the 15 plasma samples per condition) in the $8000-10000 \mathrm{~m} / \mathrm{z}$ range. The $\mathrm{H} 50$ chip-processed profiles show the relative abundance of chipbinding proteins. A boxed region identifies a cluster near 9411 $\mathrm{m} / \mathrm{z}$ ratio differentially expressed in the three groups $(p<0.05$, Mann-Whitney U-test).

protein peaks were $8674.51 \quad(p=0.0284), \quad 8789.99$ $(p=0.0334), 8909.35(p=0.0059), 9411.68(p=0.0003)$ and $9689.32(p=0.0015)$ respectively. We identified a peak of about $9411 \mathrm{Da}(z=1)$ that was highly differentially expressed in the plasma from donors who had resolved their HCV infection. A scatter plot of the normalized linear intensity of the candidate $9.4 \mathrm{kDa}$ marker in all plasma is shown in Fig. 2 and the mean intensities of cases within each group are indicated by the bar. The mean intensities \pm SD are as follows: $4.78 \pm 1.93$ (Negative), $3.03 \pm 0.77$ (Resolved) and $2.29 \pm 0.90$ (Chronic) respectively. Table 2 shows the $p$ values for the mean intensities of the $9.4 \mathrm{kDa}$ marker for pairwise comparisons between the different sample groups. The mean intensity of the $9.4 \mathrm{kDa}$ marker was higher in negative donors than in donors who had resolved their HCV 


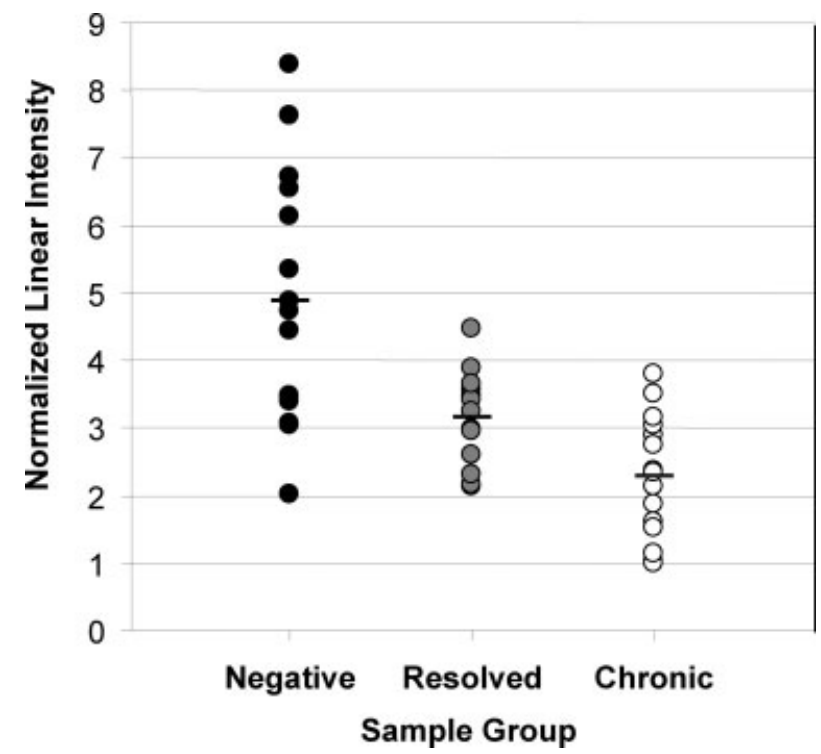

Figure 2. Distribution of the normalized linear intensity of the $9.4 \mathrm{kDa}$ marker of each sample in all of the sample groups. Biomarker Wizard generated normalized linear intensity. SoftwareBar indicates the mean intensity of all samples within each group.

Table 2. Comparisons of means (Wilcoxon test) between the groups of donors for the $9.4 \mathrm{kDa}$ marker

\begin{tabular}{ll}
\hline Group pairs & $p$-value \\
\hline Negative vs. Resolved & 0.01141 \\
Negative vs. Chronic & 0.00022 \\
Resolved vs. Chronic & 0.02910 \\
\hline
\end{tabular}

infection $(p=0.0114)$ or in donors with chronic HCV infection $(p=0.0002)$. The mean intensity of this marker was higher in donors who had resolved their HCV infection than in donors with chronic HCV infection $(p=0.0291)$. This $9.4 \mathrm{kDa}$ polypeptide may be considered as a candidate marker associated with the resolution of HCV infection.

\subsection{Enrichment of the $9.4 \mathrm{kDa}$ candidate marker}

We developed a salting-out strategy for enrichment and purification of the candidate marker. Plasma fractionation was performed using increased concentrations of salt to reach 20 , $40,60,80$ and $100 \%$ saturation of ammonium sulfate. The precipated proteins obtained from the plasma of a donor who had resolved his HCV infection were analyzed by 1-D SDSPAGE. As shown in Fig. 3a, two bands ( $a$ and $b$ ) migrating with an apparent mass near $9.4 \mathrm{kDa}$ were visualized on the CBB stained gel in the $80 \%$ saturation fraction but not in the original unfractionated plasma. A control by SELDI-TOF-MS was performed on a normal phase NP20 array and confirmed the enrichment of the $9.4 \mathrm{kDa}$ marker in the $80 \%$ saturation fraction (Fig. 3b). The two bands of interest were then excised for passive elution and were analyzed by SELDI-TOF-MS on a H50 array. Figure $3 \mathrm{c}$ depicts the SELDI-TOF-MS spectra for each eluted protein from the $b$ band, demonstrating that one protein gave a singly charged $9.4 \mathrm{kDa}$ peak similar to the candidate protein identified in the original profiling study.

\subsection{Identification of the 9.4 kDa candidate marker}

The $b$ band migrating on SDS-PAGE with an apparent $9.4 \mathrm{kDa}$ mass was excised, digested with trypsin and analyzed by nanoflow-HPLC-Q-TOF-MS/MS. Three peptides pointed to the identification of ApoC-III (Swiss-Prot accession No P02656) (Fig. 4 a, b and c). Ions with $m / z$ of 572.97 $\mathrm{MH}^{3+}, 858.95 \mathrm{MH}^{2+}$ and $449.74 \mathrm{MH}^{2+}$ were identified as a fragments of ApoC-III with MASCOT probability score of 316 (Fig. 4 d) and a 33\% sequence coverage of the secreted form. This form of ApoC-III corresponds to the mature secreted form with signal peptide cleaved. We purchased purified ApoC-III protein and analyzed the SELDI-TOF-MS profil on a NP20 array (Fig. 4e). Pure ApoC-III resulted in three peaks at $9153.5,9443.1$ and $9730.8 \mathrm{~m} / \mathrm{z}$ ratios corresponding to the three isoforms termed as ApoC-III 0 , ApoC$\mathrm{III}_{1}$ and ApoC-III $\mathrm{I}_{2}$ that have been shown to contribute, respectively, to approximately 10,55 and $35 \%$ of the total ApoC-III levels in circulation [23].

\subsection{Validation of identified ApoC-III}

Identity of ApoC-III was validated by western blot analysis and by immunoturbidimetric assay. Western blot analysis using a specific rabbit anti-human ApoC-III antibody detected ApoC-III in unfractionated human plasma and in the $80 \%$ saturation fraction (Fig. 5). A heavier band at approximately $20 \mathrm{kDa}$ was also observed for the purified ApoC-III as specified by the manufacturer.

To further confirm the western blot result, ApoC-III levels were measured by immunoturbidimetric assay on the 45 human plasma samples previously described. Differential expression of ApoC-III was validated by the significant decrease in ApoC-III levels observed when comparing the Negative group $(0.088 \pm 0.032 \mathrm{~g} / \mathrm{L})$ to the Resolved group $(0.053 \pm 0.019 \mathrm{~g} / \mathrm{L})$ or to the Chronic group $(0.040 \pm$ $0.001 \mathrm{~g} / \mathrm{L}$ ) (Fig. 6). The $p$-values for the mean concentrations of ApoC-III for pairwise comparisons between the different sample groups are indicated by a bar (Fig. 6). However, no obvious clinical correlation was found between the ApoC-III levels and the HCV viral loads (data not shown). The results from immunoassay are consistent with SELDI-TOF-MS data.

\subsection{Plasma lipid parameters}

The comparison of plasma lipid profiles between unexposed donors, donors with resolved HCV infection and with chronic HCV infection confirmed the key role for ApoC-III 
a)

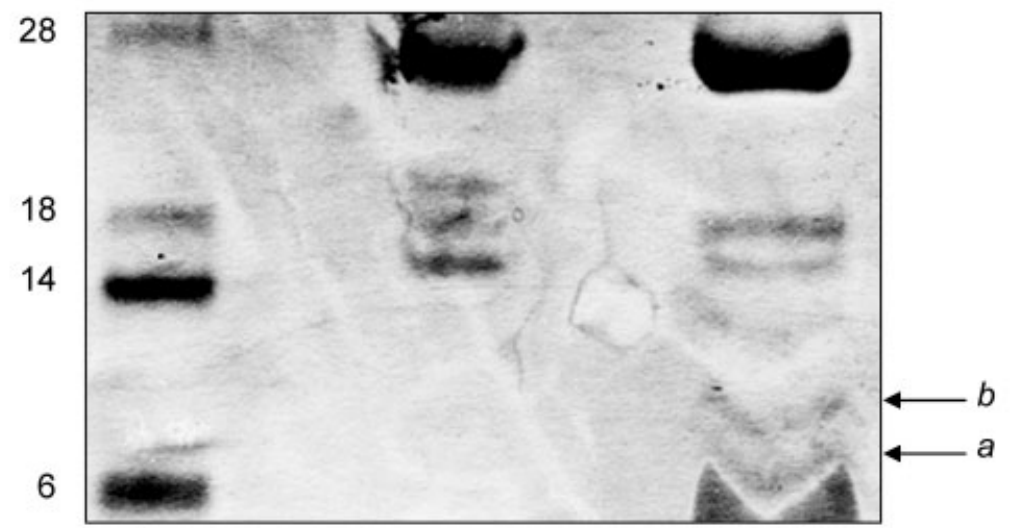

b)

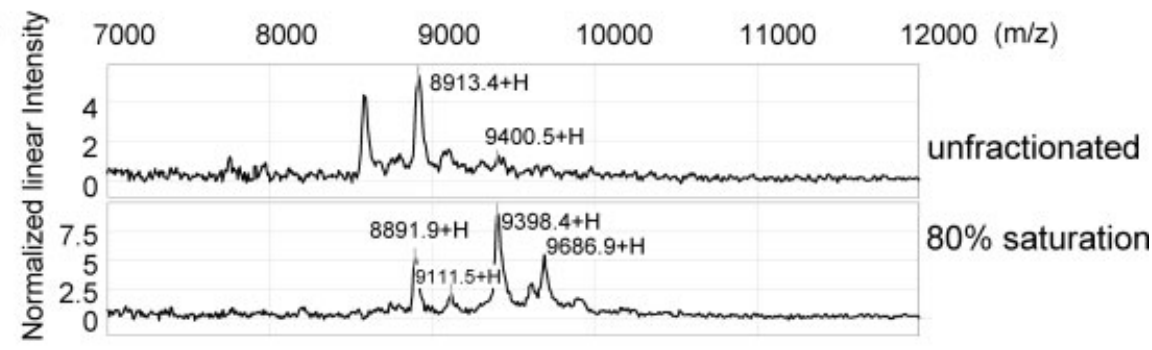

c)

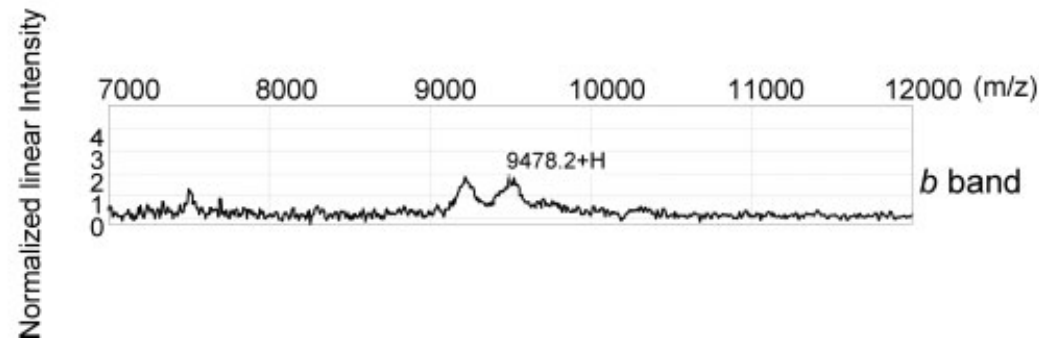

Figure 3. Results of the enrichment process. (a) SDS-PAGE analysis of both unfractionated plasma from a donor with resolved $\mathrm{HCV}$ infection and of one enriched fraction obtained by salting out with $80 \%$ saturation of ammonium sulfate. The separated proteins were stained with CBB. Molecular weight protein markers are indicated. (b) Confirmation of enrichment of the $9.4 \mathrm{kDa}$ marker by SELDITOF-MS analysis on NP20 array in unfractionated plasma and enriched fraction respectively. Gel bands $a$ and $b$ from the enriched fraction were excised and subjected to passive elution. (c) The proteins eluted from the $b$ band bound to H50 array and SELDI-TOF-MS analysis of the spectra indicate a $9.4 \mathrm{kDa}$ peak. as this protein is the only one which shows a statistically different expression between the three groups (Table 3). Indeed decreased ApoC-III levels in the Resolved group were not linked to variations in other measured apolipoproteins (ApoA-I, ApoA-II, Apo B, ApoC-I, ApoC-II, ApoE), nor in total cholesterol, HDL-cholesterol, LDL-cholesterol, VLDL-cholesterol and TG levels when compared to the Negative group. By contrast, the Chronic group exhibited a significant lipid metabolism impairment when compared to the Negative group. This was characterized by a decrease in total cholesterol, LDL-cholesterol and TG levels, associated with lower levels of ApoB, ApoC-II and ApoC-III (Table 3).

\section{Discussion}

The classical separation techniques, including 2-D PAGE, are time consuming and lead to a poor resolution of hydrophobic proteins as well as of low molecular mass polypep- tides $(<20 \mathrm{kDa})$. The tremendous complexity of the plasma proteome is paradoxically the source of both its extraordinary value in diagnosis and of great difficulties in analysis. The challenge is to detect the proteins present only in low abundances, since $99 \%$ of the protein in plasma is made up of about 20 highly abundant species. New biomarkers are expected to be found among trace proteins [10]. To circumvent these limitations, we have used SELDI-TOF-MS technology (www.bio-rad.com/proteinchip), a proteomic technique that rapidly performs the analysis of proteins at the femtomole level and provides a research platform to compare many different plasma samples.

Hepatitis C infection represents a serious health problem worldwide. The mechanisms by which HCV enters and infects host cells are incompletely understood. The LDLreceptor has been suspected to play a role in HCV infection on the basis of the well-documented interaction between HCV and lipoprotein [24-26]. LDL-receptor has been identified as a receptor candidate for HCV [27-30]. We have 
a)

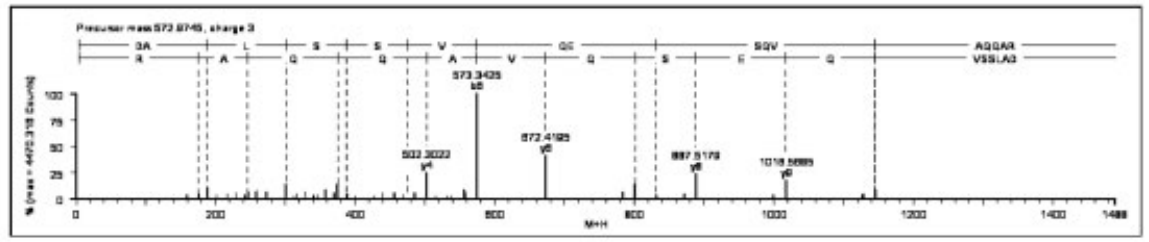

b)

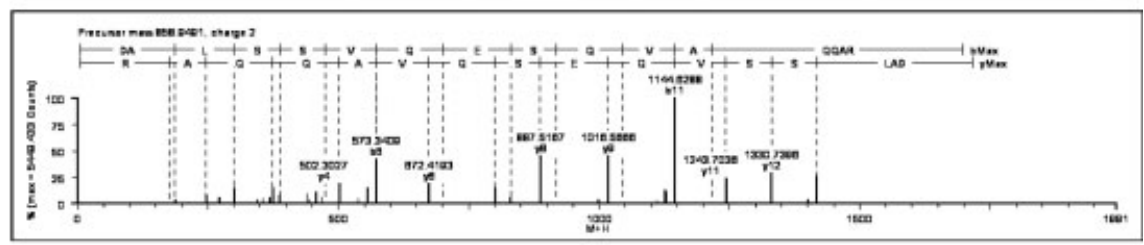

c)

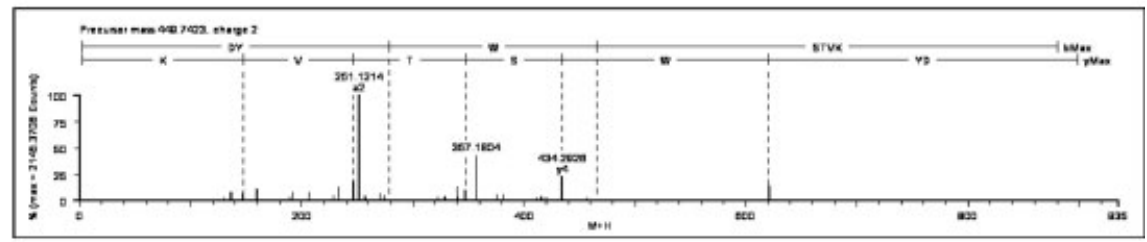

d)

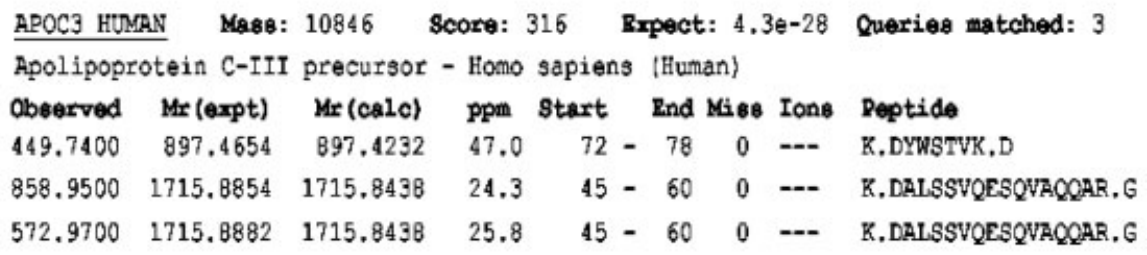

e)

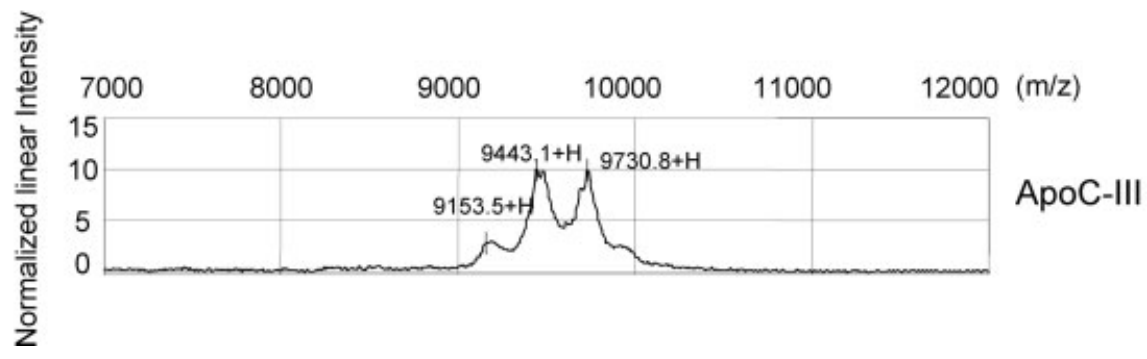

Figure 4. Identification of the $9.4 \mathrm{kDa}$ marker by nanoLC-QTOF-MS/MS. MS/MS analysis of ion with $\mathrm{m} / \mathrm{z}$ (a) $572.97 \mathrm{MH}^{3+}$, (b) $858.95 \mathrm{MH}^{2+}$ and (c) 449.74 $\mathrm{MH}^{2+}$. (d) lons were identified as tryptic fragments of ApoC-III (P02656) with probability based Mowse Score of 316. (e) SELDITOF-MS analysis on NP20 array of purified ApoC-III. recently shown that the LDL-receptor plays a role in the infection of primary human hepatocytes by HCV from human serum [31]. Other receptors including CD81 [32], the scavenger receptor class B type I (SRBI), a receptor of HDL, LDL and VLDL [33-37] and more recently Claudin-1 [38], have also been implicated in cell virus entry. In recent genomic analysis of liver biopsies from acutely infected chimpanzees and of subgenomic replicon models, the accumulation of free fatty acids associated with transcriptional changes in host genes involved in lipid metabolism was reported to have a positive effect on the HCV replicon and may have a similar effect on HCV replication [39]. Furthermore, Jacobs et al. demonstrated that transfection of hepatoma permissive Huh-7.5 cells with a full lengh HCV replicon induces several changes in protein abundance indicative of disturbances in lipid metabolism [40]. It was proposed that accumulation of free fatty acids benefits the virus. Consistent with these data, Kapadia et al. demonstrated that elements of the cholesterol and fatty-acid-biosynthetic pathways are required for HCV RNA replication in Huh-7 cells [41]. 


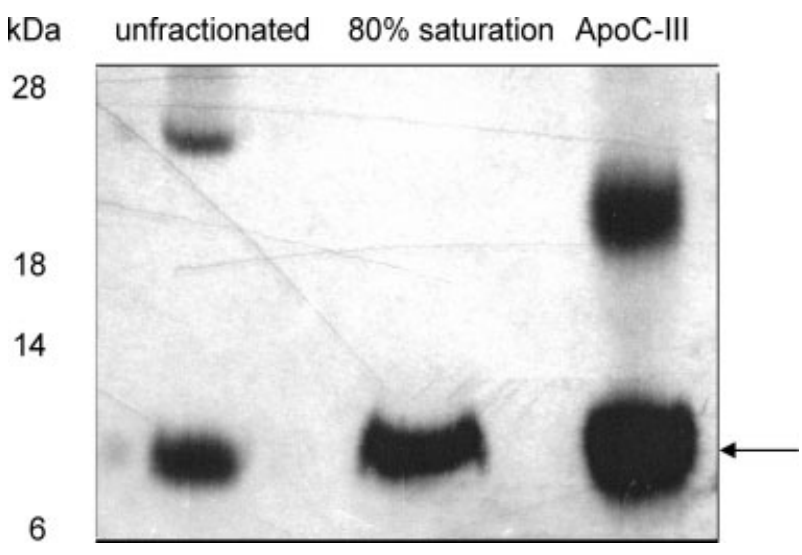

Figure 5. Western blot analysis of ApoC-III levels of both unfractionated plasma from a donor with resolved $\mathrm{HCV}$ infection and of one enriched fraction obtained by salting out with $80 \%$ saturated ammonium sulfate. A human purified ApoC-III $(2 \mu \mathrm{g})$ was used as control. ApoC-III antibody was used at a 1:20000 dilution and secondary anti-rabbit HRP at 1:10000. Molecular weight protein markers are indicated.

The accessibility of archived plasma samples in optimal storage conditions fully characterized in blood transfusion services makes these samples the ideal candidates for the identification of biomarkers for clinical studies [11, 42]. To identify candidate biomarkers associated with the resolution of HCV infection, protein expression profiles from plasma samples from blood donors who have cleared the virus, versus those from HCV negative donors and from donors with chronic hepatitis were compared by SELDI-TOF-MS. We found a $9.4 \mathrm{kDa}$ protein that was highly differentially expressed in the three groups. This peak was identified as ApoC-III and the identity was validated by western blot analysis and by immunoassay. Consistent with SELDI-TOF-MS

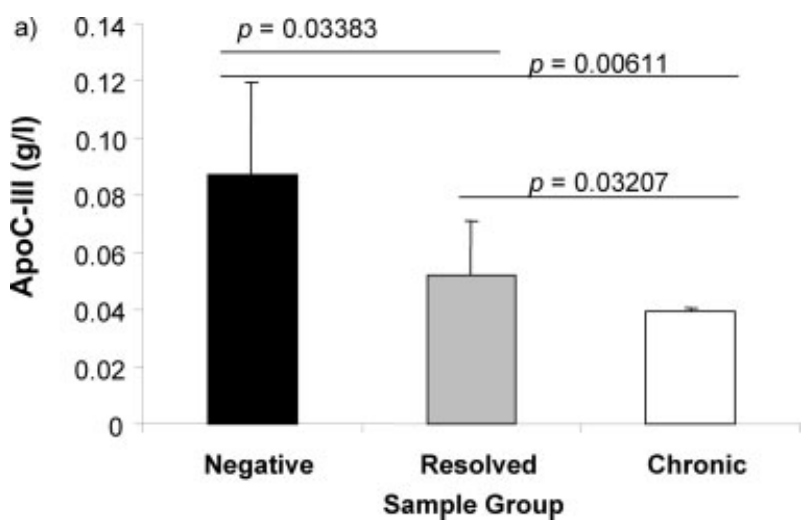

Figure 6. Plasmatic ApoC-III levels assayed by immunoturbidimetric experiments on 15 samples from negative donors, 15 samples from donors with resolved HCV infection and 15 samples from chronic carriers.

data, the mean concentration levels of ApoC-III were higher in unexposed donors than in those who had resolved their $\mathrm{HCV}$ infection and in donors with chronic HCV infection. The analysis of lipid profiles of all plasma samples showed that the ApoC-III level was the only statistically different parameter between the three groups. ApoC-III levels were significantly lower in patients with resolved infection than in unexposed donors, while no significant difference was observed in other apolipoproteins (ApoA-I, ApoA-II, ApoB, ApoC-I, ApoC-II, and ApoE), nor in cholesterol and TG concentrations, suggesting that the reduction is linked to HCV and not only to liver damage. Finally, no correlation was found between the ApoC-III values and that of the HCV viral load in chronically infected patients. This suggests that ApoC-III could play a role during the acute phase of HCV infection.

Table 3. Plasma lipid parameters

\begin{tabular}{|c|c|c|c|c|}
\hline & Negative & Resolved & Chronic & Normal Range \\
\hline ApoA-I (g/L) & $1.63 \pm 0.40$ & $1.64 \pm 0.29$ & $1.56 \pm 0.26$ & $1.10-1.80$ \\
\hline ApoA-II (g/L) & $0.38 \pm 0.08$ & $0.37 \pm 0.10$ & $0.37 \pm 0.07$ & $0.32-0.54$ \\
\hline $\operatorname{ApoB}(g / L)^{a), b)}$ & $0.93 \pm 0.20$ & $0.95 \pm 0.20$ & $0.75 \pm 0.19$ & $0.50-1.82$ \\
\hline ApoC-I (g/L) & $0.073 \pm 0.018$ & $0.079 \pm 0.014$ & $0.076 \pm 0.014$ & $0.057-0.089$ \\
\hline ApoC-II $(g / L)^{a), b)}$ & $0.032 \pm 0.017$ & $0.029 \pm 0.013$ & $0.013 \pm 0.010$ & $0.016-0.042$ \\
\hline ApoC-III $(g / L)^{a), b), c)}$ & $0.088 \pm 0.032$ & $0.053 \pm 0.019$ & $0.040 \pm 0.001$ & $0.05-0.12$ \\
\hline ApoE (g/L) & $0.038 \pm 0.015$ & $0.034 \pm 0.009$ & $0.036 \pm 0.011$ & $0.027-0.045$ \\
\hline Total cholesterol $(g / L)^{a), b)}$ & $2.10 \pm 0.42$ & $2.04 \pm 0.30$ & $1.73 \pm 0.35$ & $1.60-2.21$ \\
\hline HDL-cholesterol (g/L) & $0.66 \pm 0.29$ & $0.56 \pm 0.12$ & $0.63 \pm 0.13$ & $>0.45$ \\
\hline LDL-cholestérol $(\mathrm{g} / \mathrm{L})^{\mathrm{a}, \mathrm{b})}$ & $0.97 \pm 0.22$ & $0.97 \pm 0.24$ & $0.75 \pm 0.21$ & $<1.60$ \\
\hline VLDL-cholesterol (g/L) ${ }^{\mathrm{a})}$ & $0.46 \pm 0.22$ & $0.49 \pm 0.14$ & $0.35 \pm 0.16$ & 0.30 \\
\hline TG $(g / L)^{a), b)}$ & $1.73 \pm 0.96$ & $1.42 \pm 0.67$ & $0.94 \pm 0.34$ & $0.53-1.49$ \\
\hline
\end{tabular}

Values are mean $\pm \mathrm{SD}$. Statistics by Wilcoxon test ( $n=15$ per group)

a) $p<0.05$ : Resolved vs. Chronic

b) $p<0.05$ : Negative vs. Chronic

c) $p<0.05$ : Negative vs. Resolved 
Many proteins of the apolipoprotein groups were used as biomarkers in cancer [44-47]. ApoA-I could be a useful biomarker for HIV diagnosis [48]. ApoC-I and ApoC-III were reported as potential plasmatic markers to distinguish between ischemic and hemorragic stroke [49]. In our study, we discovered a significant variation in the average differential expression of ApoC-III between unexposed donors, donors with resolved $\mathrm{HCV}$ infection and chronic HCV carriers. These findings suggest that ApoC-III can potentially be used as a biomarker associated with the resolution of HCV infection. ApoC-III, a protein secreted mostly by the liver, is associated with both triglyceride-rich lipoproteins and HDL in peripheral circulation $[23,50,51]$. This is the most abundant apolipoprotein $\mathrm{C}$ in human plasma $[50,51]$. ApoC-III is present in three isoforms that are termed ApoC-III $\mathrm{I}_{0}$, ApoC$\mathrm{III}_{1}$ and ApoC-III ${ }_{2}$ depending on the number of sialic molecules ( 0 to 2 ) terminating the oligosaccharidic portions of the protein. Each isoform has been shown to contribute, respectively, to approximately 10, 55 and $35 \%$ of the total ApoC-III levels in circulation [23]. Rossi et al. previously described that ApoC-III isoforms should be associated to three dominant peaks in SELDI spectra with averaged $m / z$ values at 9162.31, 9411.96 and 9707.00 using strong anionic exchange chips [43]. Thus, we have analyzed the peaks obtained from our profiling study with $\mathrm{m} / z$ value at position $9126.76,9411.68$ and 9689.32 that were suspected to be associated to ApoC$\mathrm{III}_{0}$, ApoC-III ${ }_{1}$ and ApoC-III ${ }_{2}$ respectively. The comparative analysis between the mean intensities of these three peaks in each group ( $n=15$ per group) and the normal distribution in circulation showed no significant difference in the distribution of the three potential ApoC-III isoforms within each group (data not shown).

Proposed mechanisms underlying the hypertriglyceridemic effect of ApoC-III comprise inhibition of lipoprotein lipase activity, disruption of interaction of triglyceride-rich lipoproteins with vessel wall heparan sulfate proteoglycans and lower clearance of ApoB-containing lipoproteins by LDLreceptor and LDL-related receptors [52]. Previous studies have shown that ApoC-III completely abolishes the ApoBmediating binding of lipoproteins to the LDL-receptor and this inhibitory action is probably due to the masking of the receptor domain of $\mathrm{ApoB}$ by ApoC-III [50]. $\mathrm{ApoB}_{100}$, exclusively secreted by the liver, is an obligatory constituent of VLDL, HDL and LDL [53]. Interestingly, evidence suggests that the LDL-receptor, which recognizes ApoB and ApoE apolipoproteins exposed on lipoproteins, mediates the binding and endocytosis of native HCV particles isolated from patient's blood, most likely via their association with LDL or VLDL [37]. Furthermore, another report has also shown that ApoB mediates interaction of natural HCV with SRBI [34]. In addition, Andréo et al. suggested recently that lipoprotein lipase mediates HCV cell entry by a mechanism similar to hepatic clearance of triglyceride-rich lipoproteins from the circulation, promoting a non-productive virus uptake [54]. Another ligand of SRBI, serum amyloid A, has an antiviral activity against HCV [56, 57] and HIV [58]. A recent report described that ApoC-I, an exchangeable apolipoprotein that predominantly resides in HDL, increases the fusion rates between viral and target membranes via a direct interaction with HCV particles [55]. Finally, Kapadia et al. recently demonstrated that HCV infection is dependent on a cooperative interaction between CD81 and SRBI and that cellular cholesterol content has a significant impact on HCV entry in Huh-7 cells [59]. Taken together, these in vitro studies outlined the strong relationship between the lipid metabolism and the evolution of $\mathrm{HCV}$ infection also observed in vivo [24, 60-62 ].

Given the correlation between HCV infection and lipid metabolism, we can hypothesize that ApoC-III is involved in differential HCV infection evolution. Moreover, decreased ApoC-III level was previously reported in HCV infection in regard to HCV genotype [63], underlining a complex relationship between HCV infection and lipoprotein metabolism that could be dependent on other factors associated with HCV entry and the early innate immune response.

In conclusion, we have identified ApoC-III as a potential low-molecular weight plasma biomarker associated with the resolution of $\mathrm{HCV}$ infection. This work requires further investigation, in a large cohort of unexposed donors or donors with HCV clearance or chronic infection, to study the predictive value of this candidate biomarker and to determine if ApoC-III is an actor or a marker of differential HCV infection evolution. The proportion of the three isoforms of ApoC-III will be evaluated in the three groups. It is likely that lipoproteins analyses in this large cohort of patients will be helpful in furthering the understanding of the basic processes promoting spontaneous HCV clearance.

We thank Laurent Tiers (Hôpital St Eloi, Montpellier) and Myriam Cubizolles (Ciphergen Ltd France) for helpful discussions on SELDI-TOF-MS technology and Jean-paul Pais de Barros for ApoC-I analysis. This study was supported by grants from Agence Nationale de Recherches sur le SIDA et les Hépatites virales (ANRS).

The authors have declared no conflict of interest.

\section{References}

[1] Chisari, F. V., Unscrambling hepatitis C virus-host interactions. Nature 2005, 436, 930-932.

[2] Thimme, R., Oldach, D., Chang, K. M., Steiger, C. et al., Determinants of viral clearance and persistence during acute hepatitis C virus infection. J. Exp. Med. 2001, 194, 1395-1406.

[3] Bowen, D. G., Walker, C. M., Adaptive immune responses in acute and chronic hepatitis C virus infection. Nature 2005, 436, 946-952.

[4] Orland, J. R., Wright, T. L., Cooper, S., Acute hepatitis C. Hepatology 2001, 33, 321-327. 
[5] Lauer, G. M., Walker, B. D., Hepatitis C virus infection. N. Engl. J. Med. 2001, 345, 41-52.

[6] Houghton, M., Abrignani, S., Prospects for a vaccine against the hepatitis C virus. Nature 2005, 436, 961-966.

[7] Busch, M. P., Insights into the epidemiology, natural history and pathogenesis of hepatitis $C$ virus infection from studies of infected donors and blood product recipients. Transfus. Clin. Biol. 2001, 8, 200-206.

[8] Busch, M. P., Glynn, S. A., Stramer, S. L., Orland, J. et al., Correlates of hepatitis $\mathrm{C}$ virus (HCV) RNA negativity among HCV-seropositive blood donors. Transfusion 2006, 46, 469475.

[9] Mosley, J.W., Operskalski, E.A., Tobler, L.H., Andrews, W.W. et al., Correlates of hepatitis C virus (HCV) RNA negativity among HCV-seropositive blood donors. Hepatology 2005, $42,86-92$.

[10] Lathrop, J. T., Hayes, T. K., Carrick, K., Hammond, D. J., Rarity gives a charm: evaluation of trace proteins in plasma and serum. Expert Rev. Proteomics 2005, 2, 393-406.

[11] Aldred, S., Grant, M. M., Griffiths, H. R., The use of proteomics for the assessment of clinical samples in research. Clin. Biochem. 2004, 37, 943-952.

[12] Issaq, H. J., Conrads, T. P., Prieto, D. A., Tirumalai, R., Veenstra, T. D., SELDI-TOF MS for diagnostic proteomics. Anal. Chem. 2003, 75,148A-155A.

[13] Tang, N., Tornatore, P., Weinberger, S. R., Current developments in SELDI affinity technology. Mass Spectrom. Rev. 2004, 23, 34-44.

[14] Poon, T. C., Yip, T. T., Chan, A. T., Yip, C. et al., Comprehensive proteomic profiling identifies serum proteomic signatures for detection of hepatocellular carcinoma and its subtypes. Clin. Chem. 2003, 49, 752-760.

[15] Paradis, V., Degos, F., Dargere, D., Pham, N. et al., Identification of a new marker of hepatocellular carcinoma by serum protein profiling of patients with chronic liver diseases. Hepatology 2005, 41, 40-47.

[16] Ward, D. G., Cheng, Y., N'Kontchou, G., Thar, T. T. et al., Changes in the serum proteome associated with the development of hepatocellular carcinoma in hepatitis C-related cirrhosis. Br. J. Cancer 2006, 94, 287-292.

[17] Schwegler, E. E., Cazares, L., Steel, L. F., Adam, B. L. et al., SELDI-TOF MS profiling of serum for detection of the progression of chronic hepatitis $C$ to hepatocellular carcinoma. Hepatology 2005, 41, 634-642.

[18] Lee, I. N., Chen, C. H., Sheu, J. C., Lee, H. S. et al., Identification of complement C3a as a candidate biomarker in human chronic hepatitis C and HCV-related hepatocellular carcinoma using a proteomics approach. Proteomics 2006, 6, 2865-2873.

[19] Englard, S., Seifter, S., Precipitation techniques. Methods Enzymol. 1990, 182, 285-300.

[20] Jiang, L., He, L., Fountoulakis, M., Comparison of protein precipitation methods for sample preparation prior to proteomic analysis. J. Chromatogr. A 2004, 1023, 317-320.

[21] Currid, C. A., O'Connor, D. P., Chang, B. D., Gebus, C. et al., Proteomic analysis of factors released from p21-overexpressing tumour cells. Proteomics 2006, 6, 3739-3753.

[22] Dautin, G., Soltani, Z., Ducloux, D., Gautier, T. et al., Hemodialysis reduces plasma apolipoprotein $\mathrm{C}$-I concentration making VLDL a better substrate for lipoprotein lipase. Kidney Int. 2007, 72, 871-878.

[23] Mauger, J. F., Couture, P., Bergeron, N., Lamarche, B., Apolipoprotein $\mathrm{C}$-III isoforms: kinetics and relative implication in lipid metabolism. J. Lipid Res. 2006, 47, 1212-1218.

[24] Andre, P., Komurian-Pradel, F., Deforges, S., Perret, M. et al., Characterization of low- and very-low-density hepatitis C virus RNA-containing particles. J. Virol. 2002, 76, 6919-6928.

[25] Kanto, T., Hayashi, N., Takehara, T., Hagiwara, H. et al., Density analysis of hepatitis $\mathrm{C}$ virus particle population in the circulation of infected hosts: implications for virus neutralization or persistence. J. Hepatol. 1995, 22, 440-448.

[26] Thomssen, R., Bonk, S., Thiele, A., Density heterogeneities of hepatitis $C$ virus in human sera due to the binding of betalipoproteins and immunoglobulins. Med. Microbiol. Immunol. 1993, 182, 329-334.

[27] Agnello, V., Abel, G., Elfahal, M., Knight, G. B., Zhang, Q. X., Hepatitis $C$ virus and other flaviviridae viruses enter cells via low density lipoprotein receptor. Proc. Natl. Acad. Sci. U S A 1999, 96, 12766-12771.

[28] Monazahian, M., Bohme, I., Bonk, S., Koch, A. et al., Low density lipoprotein receptor as a candidate receptor for hepatitis C virus. J. Med. Virol. 1999, 57, 223-229.

[29] Triyatni, M., Saunier, B., Maruvada, P., Davis, A. R. et al., Interaction of hepatitis $C$ virus-like particles and cells: a model system for studying viral binding and entry. J. Virol. 2002, 76, 9335-9344.

[30] von Hahn, T., McKeating, J. A., In vitro veritas? The challenging of studying hepatits $C$ virus infectivity in a test tube. $J$. Hepatol. 2007, 46, 355-358.

[31] Molina, S., Castet, V., Fournier-Wirth, C., Pichard-Garcia, L. et al., The low-density lipoprotein receptor plays a role in the infection of primary human hepatocytes by hepatitis $\mathrm{C}$ virus. J. Hepatol. 2007, 46, 411-419.

[32] Pileri, P., Uematsu, Y., Campagnoli, S., Galli, G. et al., Binding of hepatitis C virus to CD81. Science 1998, 282, 938-941.

[33] von Hahn, T., Lindenbach, B. D., Boullier, A., Quehenberger, O. et al., Oxidized low-density lipoprotein inhibits hepatitis $C$ virus cell entry in human hepatoma cells. Hepatology 2006, 43, 932-942.

[34] Maillard, P., Huby, T., Andreo, U., Moreau, M. et al., The interaction of natural hepatitis $C$ virus with human scavenger receptor SR-BI/Cla1 is mediated by ApoB-containing lipoproteins. FASEB J. 2006, 20, 735-737.

[35] Scarselli, E., Ansuini, H; Cerino, R., Roccasecca, R. M. et al., The human scavenger receptor class $B$ type $I$ is a novel candidate receptor for the hepatitis C virus. EMBO J. 2002, 21, 5017-5025.

[36] Voisset, C., Callens, N., Blanchard, E., Op De Beeck, A. et al., High density lipoproteins facilitate hepatitis $C$ virus entry through the scavenger receptor class B type I. J. Biol. Chem. 2005, 280, 7793-7799.

[37] Dreux, M., Pietschmann, T., Granier, C.,Voisset, C. et al., High density lipoprotein inhibits hepatitis $C$ virus-neutralizing antibodies by stimulating cell entry via activation of the scavenger receptor BI. J. Biol. Chem. 2006, 281, 1828518295.

[38] Evans, M. J., von Hahn, T., Tscherne, D. M., Syder, A. J. et al., Claudin- 1 is a hepatitis $C$ virus co-receptor required for a late step in entry. Nature 2007, 446, 801-805. 
[39] Su, A. I., Pezacki, J. P., Wodicka, L. , Brideau, A. D. et al., Genomic analysis of the host response to hepatitis $C$ virus infection. Proc. Natl. Acad. Sci. U S A 2002, 99, 15669-15674.

[40] Jacobs, J. M., Diamond, D. L., Chan, E. Y.,Gritsenko, M. A. et al., Proteome analysis of liver cells expressing a full-length hepatitis $\mathrm{C}$ virus (HCV) replicon and biopsy specimens of posttransplantation liver from HCV-infected patients. J. Virol. 2005, 79, 7558-7569.

[41] Kapadia, S. B., Chisari, F. V., Hepatitis C virus RNA replication is regulated by host geranylgeranylation and fatty acids. Proc. Natl. Acad. Sci. U S A 2005, 102, 2561-2566.

[42] Hsieh, S. Y., Chen, R. K., Pan, Y. H., Lee, H. L., Systematical evaluation of the effects of sample collection procedures on low-molecular-weight serum/plasma proteome profiling. Proteomics 2006, 6, 3189-3198.

[43] Rossi, L., Martin, B. M., Hortin, G. L., White, R. L. et al., Inflammatory protein profile during systemic high dose interleukin-2 administration. Proteomics 2006, 5, 709-720.

[44] Kozak, K. R., Su, F., Whitelegge, J. P., Faull, K. et al., Characterization of serum biomarkers for detection of early stage ovarian cancer. Proteomics 2005, 5, 4589-4596.

[45] Ehmann, M., Felix, K., Hartmann, D., Schnolzer, M. et al., Identification of potential markers for the detection of pancreatic cancer through comparative serum protein expression profiling. Pancreas 2007, 34, 205-214.

[46] Zhang, Z., Bast, R. C., Jr., Yu, Y., Li, J. et al., Three biomarkers identified from serum proteomic analysis for the detection of early stage ovarian cancer. Cancer Res. 2004, 64, 58825890.

[47] Malik, G., Ward, M. D., Gupta, S. K., Trosset, M. W. et al., Serum levels of an isoform of apolipoprotein A-II as a potential marker for prostate cancer. Clin. Cancer Res. 2005, 11, 1073-1085.

[48] Kim, S. S., Kim, M. H., Shin, B. K. , Na, H. J. et al., Different isoforms of apolipoprotein Al present in heterologous posttranslational expression in HIV infected patients. J. Proteome Res. 2007, 6, 180-184.

[49] Allard, L., Lescuyer, P., Burgess, J., Leung, K. Y. et al., ApoC-I and ApoC-III as potential plasmatic markers to distinguish between ischemic and hemorrhagic stroke. Proteomics 2004, 4, 2242-2251.

[50] Jong, M. C., Hofker, M. H., Havekes, L. M., Role of ApoCs in lipoprotein metabolism: functional differences between ApoC1, ApoC2, and ApoC3. Arterioscler. Thromb. Vasc. Biol. $1999,19,472-484$.
[51] Nestel, P. J., Fidge, N. H., Apoprotein C metabolism in man. Adv. Lipid Res. 1982, 19, 55-83.

[52] Shachter, N. S., Apolipoproteins C-I and C-III as important modulators of lipoprotein metabolism. Curr. Opin. Lipidol. 2001, 12, 297-304.

[53] Mahley, R. W., Innerarity, T. L., Rall, S.C., Jr., Weisgraber, K. $\mathrm{H} .$, Plasma lipoproteins: apolipoprotein structure and function. J. Lipid. Res. 1984, 25, 1277-1294.

[54] Andreo, U., Maillard, P., Kalinina, O., Walic, M. et al., Lipoprotein lipase mediates hepatitis $\mathrm{C}$ virus (HCV) cell entry and inhibits HCV infection. Cell. Microbiol. 2007, 9, 2445-2456.

[55] Dreux, M., Boson, B., Ricard-Blum, S., Molle, J. et al., The exchangeable apolipoprotein APOC-I promotes membrane fusion of hepatitis C virus. J. Biol., Chem. 2007, 282, 3235732369.

[56] Lavie, M., Voisset, C., Vu-Dac, N., Zurawski, V. et al., Serum amyloid $A$ has antiviral activity against hepatitis $C$ virus by inhibiting virus entry in a cell culture system. Hepatology 2006, 44, 1626-1634.

[57] Cai, Z., Cai, L., Jiang, J., Chang, K. S. et al., Human serum amyloid $A$ protein inhibits hepatitis $C$ virus entry into cells. J. Virol. 2007, 81, 6128-6133.

[58] Misse, D., Yssel, H., Trabattoni, D., Oblet, C. et al., IL-22 participates in an innate anti-HIV-1 host-resistance network through acute-phase protein induction. J. Immunol. 2007, 178, 407-415.

[59] Kapadia, S. B., Barth, H., Baumert, T., McKeating, J. A., Chisari, F. V. et al., Initiation of hepatitis $C$ virus infection is dependent on cholesterol and cooperativity between CD81 and scavenger receptor B type I. J. Virol. 2007, 81, 374-383.

[60] Wozniak, M. A., Itzhaki, R. F., Faragher, E. B., James, M. W. et al., Apolipoprotein E-epsilon 4 protects against severe liver disease caused by hepatitis C virus. Hepatology 2002, 36, 456-463.

[61] Akuta, N., Suzuki, F., Kawamura, Y.,Yatsuji, H. et al., Predictive factors of early and sustained responses to peginterferon plus ribavirin combination therapy in Japanese patients infected with hepatitis $C$ virus genotype $1 \mathrm{~b}$ : amino acid substitutions in the core region and low-density lipoprotein cholesterol levels. J. Hepatol. 2007, 46, 403-410.

[62] Negro, F., Mechanisms and significance of liver steatosis in hepatitis C virus infection. World J. Gastroenterology 2006, $14,6756-6765$.

[63] Moriya, K., Shintani, Y., Fujie, H., Miyoshi, H. et al., Serum lipid profile of patients with genotype $1 \mathrm{~b}$ hepatitis $C$ viral infection in Japan. Hepatol. Res. 2003, 25, 371-376. 